\title{
Analysis of ionospheric parameters during Solar events and ge- omagnetic storms
}

\author{
Oksana Mandrikova ${ }^{1, \star}$, Yury Polozov ${ }^{1}$, Igor Solovev ${ }^{1}$, and Nadezhda Fetisova ${ }^{1}$ \\ ${ }^{1}$ Institute of Cosmophysical Researches and Radio Wave Propagation FEB RAS, \\ Paratunka, Kamchatsky Kray, Russia
}

\begin{abstract}
The paper shows new methods of analysis of ionospheric and magnetic data applying the models of multicomponent constructions (MCM models) developed by the authors. Based on ground station data, the analysis of ionospheric and magnetic data during increased solar activity was carried out.
\end{abstract}

\section{Introduction}

During increased solar activity, anomalous changes characterizing the processes in the Earth magnetosphere and ionosphere may be observed in the recorded ionospheric parameters [1-3]. The anomalous changes manifest themselves as significant deviations (increase or decrease) of electron concentration in relation to the characteristic level. The times of occurrence, relative values and duration of ionospheric anomalies depend on many factors. In seismically active regions of the Earth, ionospheric anomalies may also be determined by increased seismic activity [1, 4]. In spite of the active development of technologies for monitoring of the near-Earth space and methods for data analysis, capabilities of estimation and forecast of ionosphere state are quite limited at the present time. Thus, development of empirical methods and technical means for their detection is of great scientific and applied significance $[1,5]$.

The problems of analysis of ionosphere state and detection of anomalies are solved by many authors [2, 3, 5-8]. Among the general approaches we can emphasize the traditional moving median method [1,9], empirical models of the ionosphere [2, 3, 5-7], application of neural networks $[2,8,10]$, and wavelet transform [10-14]. The most developed empirical model of the ionosphere is the International Reference Ionosphere (IRI) model [5], which is based on a wide range of ground and space data. The IRI accuracy for estimation of the parameters in a certain region much depends on data availability and may give results with large deviations from experimental data [5]. Thus, a more accurate forecast based on the IRI model is obtained for midlatitueds in comparison to equatorial and auroral latitudes. It was shown in the papers $[2,7]$ that the IRI model accuracy much depends on the level of solar activity and goes down as the latter grows. Compared to the IRI model, the new developments of empirical models applying neural networks $[2,8]$ allow us to improve significantly the quality of forecast. They are easily realized in automatic mode and are quite flexible. However, these models refer to the class of "black box" models, so to describe an attribute space they require

^e-mail: oksanam1@mail.ru 
long learning samples prone to overfitting and can show unexpected results in case of very noisy data. In the paper, we use a complex approach based on wavelet transform methods and their combinations with classical autoregressive approaches and neural networks. In the previous papers [4, 12-14] we showed that application of classical autoregressive methods [15] in combination with modern methods for pattern recognition allow us to obtain quite exact estimates and they are easily realized in automatic mode. The main advantage of the suggested approach is the mathematical validity and, as a consequence, the possibility to receive the results with defined confidence probability.

Ionosphere parameters were analyzed together with geomagnetic field data (www.ikir.ru). Methods described in the paper [16] were used for processing. The obtained results were compared with solar wind parameters (http://www.srl.caltech.edu/ACE/ASC).

\section{Description of methods}

\section{MCM model-1 of ionospheric parameter time series based on wavelets and autoregressive models.}

Step 1. On the basis of multiple-scale decompositions (MSD), the time series $f_{o}$ is presented in the form of a linear combination of different-scale components: smoothed one $f\left[2^{-m} t\right]$ of the scale $m$ and detailing ones $g\left[2^{j} t\right]$ of the scales $j=\overline{-1,-m}[4]$ :

$$
f_{o}(t)=\sum_{j=-1}^{-m} g\left[2^{j} t\right]+f\left[2^{-m} t\right],
$$

where $g\left[2^{j} t\right]=\sum_{n} d_{j, n} \Psi_{j, n}(t), d_{j, n}=\left\langle f, \Psi_{j, n}\right\rangle, \quad \Psi$ is the basic wavelet, $j$ is resolution, $f\left[2^{-m} t\right]=$ $\sum_{k} c_{-m, n} \phi_{-m, n}(t), \quad c_{-m, n}=\left\langle f, \phi_{-m, n}\right\rangle, \quad \phi$ is the smoothing scaling function. The inferior index 0 corresponds to the data initial resolution.

Step 2. To obtain the components of the series $f\left[2^{-m} t\right], m=\overline{1, M}$, we identify autoregressive models (the procedure of identification is described in detail in the papers [12, 14]).

Step 3. We join the obtained models into a multicomponent construction (MCM model-1) describing data time variation:

$$
f_{o}(t)=\sum_{\mu=\overline{1, T}} \sum_{k=\overline{1, N_{j}^{\mu}}} s_{j, k}^{\mu} b_{j, k}^{\mu}(t),
$$

where $s_{j, k}^{\mu}=\sum_{l=1}^{p_{j}^{\mu}} \gamma_{j, l}^{\mu} \omega_{j, k-l}^{\mu}-\sum_{n=1}^{h_{j}^{\mu}} \theta_{j, n}^{\mu} a_{j, k-n}^{\mu}$ is the assessed value of $\mu$ component; $p_{j}^{\mu}, \gamma_{j, l}^{\mu}$ are order and parameters of autoregression of $\mu$ component; $h_{j}^{\mu}, \theta_{j, k}^{\mu}$ are order and parameters of the moving average of $\mu$ component; $\omega_{j, k}^{\mu}=\nabla^{\mu^{\mu}} \beta_{j, k}^{\mu}$; $\nu^{\mu}$ is the difference order of $\mu$ component; $\beta_{j, k}^{1}=c_{j, k}, \beta_{j, k}^{\mu}=$ $d_{j, k}, \mu=\overline{2, T} ; T$ is the number of the modulated components; $a_{j, k}^{\mu}$ are residual errors of the model of $\mu$ component; $N_{j}^{\mu}$ is the length of $\mu$ component; $b_{j, k}^{1}=\phi_{j, k}$ is the scaling function; $b_{j, k}^{\mu}=\Psi_{j, k}, \mu=\overline{2, T}$ is the wavelet basis of $\mu$ component.

During the anomalous behavior of ionospheric parameters, their structure will change and, as a consequence, errors of MCM models will increase. Thus, the procedure of anomaly detection is based on the following conditional test:

$$
\varepsilon_{\mu}=\sum_{q=1}^{Q_{\mu}}\left|a_{j, k+q}^{\mu}\right|>T_{\mu},
$$

where $Q_{\mu}$ is the length of prediction of the data based on the model of $\mu$ component; $a_{j, k+q}^{\mu}=s_{j, k+q}^{\mu, f a c t}-$ $s_{j, k+q}^{\mu, \text { forecast }}$ are the residual errors of the model of $\mu$ component at the point $k+q, T_{\mu}$ is a threshold value of $\mu$ component determining the presence of an anomaly. 


\section{MCM model-2 of ionospheric parameter time series base on wavelets and neural networks}

Step 1. On the bases of MSD (see presentation (1)), we obtain a smoothed component $f\left[2^{-m} t\right]$ of the series $f_{O} F 2$. To exclude the tip effect, the initial set of data is extended by mirror segments (on the basis of function mirror reflection) with the length of $2^{-m} * q$ of counts (the size of a neighborhood containing the tip effect on a scale level $-m$ is determined by the formula $h_{-j}=2^{-m} * q$, where $q$ is the size of the applied wavelet).

Step 2. We perform wavelet reconstruction of the component $f\left[2^{-m} t\right]$ to the initial resolution removing mirror segments and obtain its presentation in the form: $f_{0}(t)=\sum_{k} c_{0, k} \phi_{0, k}(t)$.

Step 3. The obtained vector of the coefficients is $\left\{c_{0, k}\right\}_{k=1}^{K}$, where $K$ is the vector length which we divide into blocks: $\left\{c_{0, k}\right\}_{k=1}^{S},\left\{c_{0, k}\right\}_{k=2}^{S+1}, \ldots,\left\{c_{0, k}\right\}_{k=K-S}^{K}$. The length of a block is $S=24$ (determined according to the diurnal variations of hourly data of $f_{O} F 2$ ). Applying the blocks, we form a neural network (the procedure of construction of a neural network is described in the paper [17]). The quality criteria of network learning is: $E_{A}=\left(\frac{1}{R} \sqrt{\sum_{r=1}^{R} e_{A, r}^{2}(l)}\right)<\varepsilon_{A}$, where $\varepsilon_{A}>0$ is a prescribed value, $e_{A, r}(l)=\hat{c}_{0, l}^{r}-c_{0, l}^{r}$ is the solution error at discrete instant of time $l$ with the time of prediction $r, c_{0, l}^{r}$ is the desired output value and $\hat{c}_{0, l}^{r}$ is the real output value of the network, $R$ is the length of the output vector of the network. Application of a neural network allows us to reproduce characteristic variations of the series $f_{O} F 2$. During anomalous changes, neural network errors will be increased and they may be detected by the following conditional test: $E_{A}=T$, where $T$ is the threshold value determining the presence of an anomaly. During the work, MCM models were developed separately for each registration site. Taking into account the seasonal changes of $f_{O} F 2$ variation, data for different seasons were modulated separately. As long as the ionosphere state is reflected on geomagnetic field, we applied ionospheric data for time intervals of relatively calm geomagnetic field (intervals within which the total one-day K-index did not exceed 18) to model $f_{O} F 2$ typical variations. To analyze ionospheric parameters in detail, we applied estimation solutions based on continuous wavelet transform [18]

$$
\left(W_{\Psi} f\right)(b, a):=|a|^{-1 / 2} \int_{-\infty}^{\infty} f(t) \Psi\left(\frac{t-b}{a}\right) d t
$$

$\Psi$ is the wavelet, $f \in L^{2}(R), a, b \in R, a \neq 0$.

If we consider the series $f_{O} F 2$ as $f$, detection of ionospheric anomalies may be based on the application of the following decision rule [12]:

$$
P_{T_{a}}\left(W_{\Psi} f_{b, a}\right)=\left\{\begin{array}{lll}
W_{\Psi} f_{b, a}, & \text { if } & \left|W_{\Psi} f_{b, a}-W_{\Psi} f_{b, a}^{\text {med }}\right| \geq T_{a} \\
0, & \text { if } & \left|W_{\Psi} f_{b, a}-W_{\Psi} f_{b, a}^{\text {med }}\right|<T_{a}
\end{array},\right.
$$

where the threshold $T_{a}=U * S t_{a}$ determines the presence of an anomaly on the scale $a$ near the point $\xi$ containing in the carrier $\Psi_{b, a}, \quad U$ is the threshold coefficient (threshold coefficient $U=2$ was used for the years of low solar activity and $U=2.5$ was used for the years with high solar activity), $S t_{a}=\sqrt{\frac{1}{\Phi-1} \sum_{u=1}^{\Phi}\left(W_{\Psi} f_{b, a}-\overline{W_{\Psi} f_{b, a}}\right)^{2}}, \quad \overline{W_{\Psi} f_{b, a}}$ and $W_{\Psi} f_{b, a}^{m e d}$ are the average value and the median determined in a moving time window of the length $\Phi$.

Anomaly intensity at a time point $t=b$ were estimated as

$$
I_{b}=\sum_{a} \frac{\left|P_{T_{a}}\left(W_{\Psi} f_{b, a}\right)\right|}{\left\|W_{\Psi} f_{b, a}\right\|_{2}}
$$


where the norm $\left\|W_{\Psi} f_{b, a}\right\|_{2}=\sqrt{\sum_{N_{a}}\left(P_{T_{a}}\left(W_{\Psi} f_{b, a}\right)\right)^{2}}, N_{a}$ is the series length on the scale $a$.
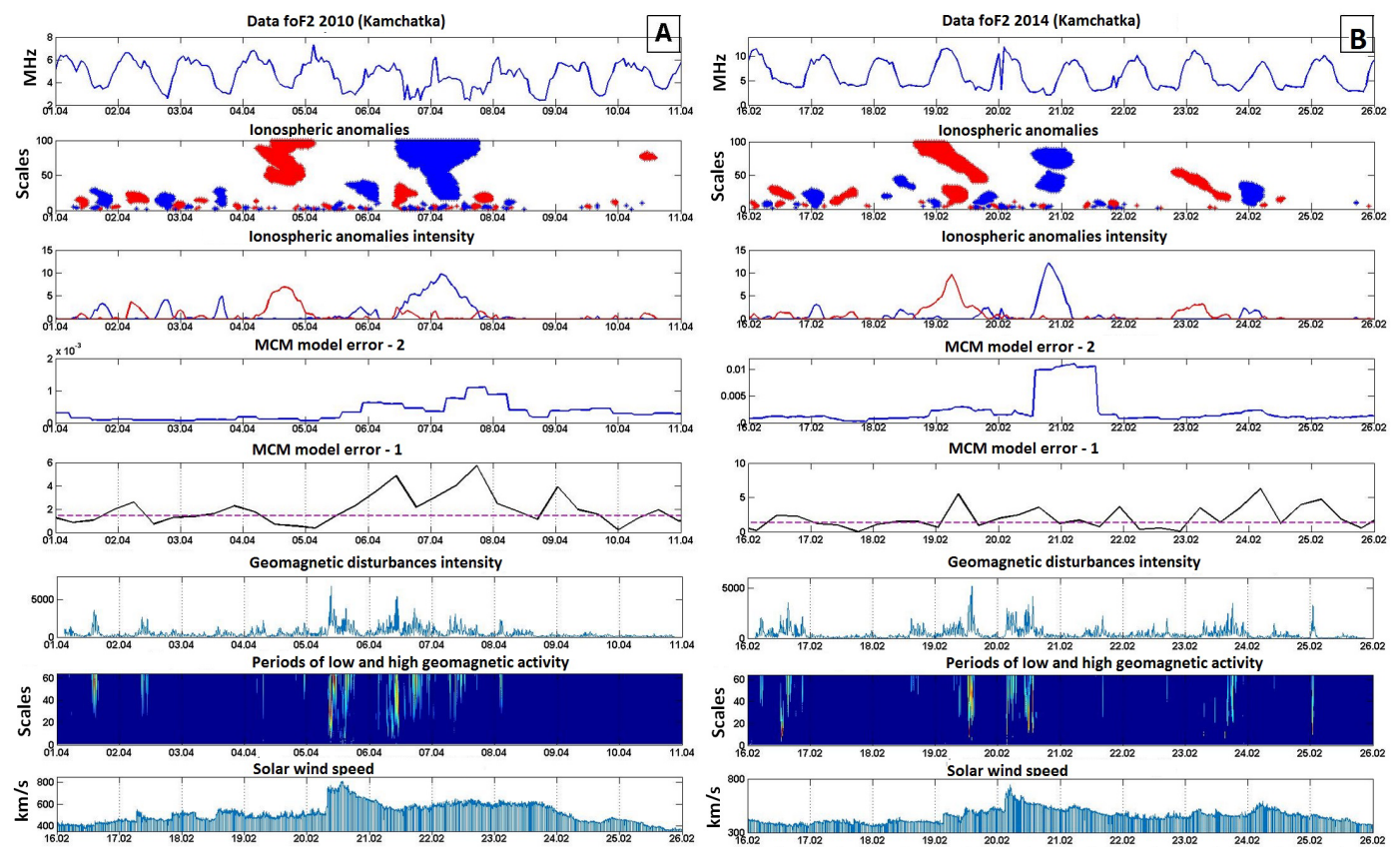

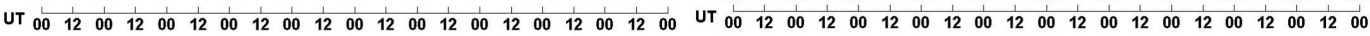

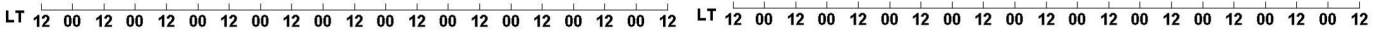

Figure 1. Results of data processing: A - April 01-10, 2010; B - February 16-26, 2014

\section{Results of data analysis}

For the processing, we applied hourly data of ionospheric parameters (www.ikir.ru) and magnetometer data (http://www.ikir.ru:8180/observ). Fig. 1 shows the results of application of the methods described above on the example of events on April 5-6, 2010 and February 19-20, 2014. A strong magnetic storm occurred within the period under analysis from April 01 to April 10, 2010 (Fig. 1A). The storm was registered on the Earth's surface at 08.26 UT as a sudden commencement (SC). According to the results of processing of ionospheric data, anomalous increases (positive phase of the ionospheric storm, Fig. 1A, marked by red color) were observed in ionospheric parameters on April 4 from 05:00 UT and to the beginning of the magnetic storm. The analysis shows that ionospheric disturbances were the most intensive in the morning (LT). During the storm, the electron concentration decreased significantly and negative phase of ionospheric storm was formed (Fig. 1A, marked by blue color) which lasted for about 30 hours. Based on the results of magnetic data processing, the strongest disturbances of geomagnetic field occurred on April 5-6 during the negative phase of the ionospheric storm. The magnetic storm which occurred on February 19-20, was registered at Paratunka (Kamchatka) site on February 19 at 12:30 UT (Fig. 1B). Anomalous increases in ionospheric parameters were observed before the event from 16:00 on February 18 till 19:00 on February 19 in Kamchatka 
region (Fig. 1B, marked by red color). Maximum values of ionospheric disturbance intensity were recorded on February 19 at 06:00 UT. By the end of the day on February 19, electron concentration decreased and negative phase of the ionospheric storm was formed which reached the highest intensity from 13:00 UT on February 20 till 04:00 UT on February 21 (Fig. 1B, marked by blue color). During anomalous behavior of the ionosphere, the highest disturbances in geomagnetic field were observed on February from 12:30 UT till 15:30 UT and on February 20 from 03:30 UT till 13:30 UT.

\section{Conclusions}

The analysis of ionosphere state showed that during increased solar activity, anomalous increases of electron density (more than 2 root mean square deviations relatively the background) may occur before storms in the regions under analysis. The detected increases which lasted from several hours to one day and a half were observed simultaneously at different stations in the North-East of Russia that characterized their large space-time scales. At present, special attention is given to the detection of ionospheric anomalies $[19,20]$. The analysis carried out in this work confirms the assumption made in the review [20] that such ionospheric effects are associated with some channel of energy penetration from the interplanetary space and magnetosphere and have solar origin. In this case, the effects may indicate an oncoming geomagnetic storm that is important for space weather forecast $[19,20]$. The methods of analysis suggested in the paper showed their efficiency in detection of such effects. The paper was supported by the Russian Scientific Foundation (grant 14-11-00194). We would like to thank the Institutes supporting the stations of cosmic ray registration and magnetic observatories which data were used in the investigations.

\section{References}

[1] E. L. Afraimovich et al., Earth, Planets and Space, 52:11, 1061-1066 (2000)

[2] E. Nakamura, T. Maruyama, Y. Shidama, Journal of the National Institute of Information and Communications Technology, 56, 391-406 (2000)

[3] K. Watthanasangmechai et al., Earth, Planets and Space, 64, 473-483 (2012)

[4] O. V. Mandrikova, N. V. Glushkova, I. V. Zhivet?ev, Geomagnetism and Aeronomy, 54:5, 638645 (2014)

[5] D. Bilitza, B. W. Reinisc, Advances in Space Research, 42, 599-609 (2008)

[6] M. V. Klimenko, Earth, Planets and Space, 64:6, 433-440 (2012)

[7] O. S. Oyekola, P. R. Fagundes, Earth, Planets and Space, 64:6, 553-566 (2012)

[8] X. Zhao et al., Advances in Space Research, 53:3, 387-394 (2012)

[9] Y. Kakinami et al., International Journal of Remote Sensing, 31:13, 3571-3578 (2010)

[10] O. V. Mandrikova et al.,A Journal of Software Engineering and Applications, 5:12B, 181-187 (2012)

[11] H. Kato et al., Journal of the National Institute of Information and Communications Technology, 56, 465-474 (2009)

[12] O. V. Mandrikova et al., Earth, Planets and Space, 67, (2015)

[13] O. V. Mandrikova, N. V. Glushkova, Yu. A. Polozov, Pattern Recognition and Image Analysis, 25:3, 470-480 (2015)

[14] O. V. Mandrikova et al., Annals of geophysics, 58:5, (2015)

[15] G. E. P. Box, G. M. Jenkins Time Series Analysis: Forecasting and Control (Holden-Day, San Francisco, 1970), 537 
[16] O. V. Mandrikova, I. S. Solovev, T. L. Zalyaev, Earth, Planets and Space, 66, (2014)

[17] O. V. Mandrikova, Yu. A. Polozov, Tsifrovaya obrabotka signalov, 2, 29-35 (2012), (in Russian)

[18] S. Mallat, A wavelet tour of signal processing (Academic Press, London, 1999), 637

[19] D. Buresova, J. Laštovička, Advances in Space Research, 39, 1298-1303 (2007)

[20] A. D. Danilov, Advances in Space Research, 52:3, 343-366 (2013) 